\title{
ON A CONJECTURE OF G. BOL
}

\author{
FR. FABRICIUS-BJERRE
}

1. It is known that a plane cubic without double points or cusps has three sextactic points lying on the branch of odd order such that each of them is the point of contact with the tangent through a point of inflection. G. Bol writes that the question whether any closed curve of odd order without double points or cusps has at least three sextactic points has not been settled [1, p. 43]. We shall prove that the answer to the question is positive, i.e., any such curve (of class $C^{4}$ ) whose singular points are ordinary points of inflection has in fact at least three sextactic points. The result is included in a more general theorem on the number of sextactic points on a closed or open curve in the projective plane.

2. Let $\gamma$ denote a curve of class $C^{4}$ lying in a projective plane and given by a parametric equation

$$
x(t)=\left(x_{0}(t), x_{1}(t), x_{2}(t)\right) .
$$

A point $P=P(t)$ is called a regular or a convex point if the determinant

$$
D(t)=\left[\boldsymbol{x} \boldsymbol{x}^{\prime} \boldsymbol{x}^{\prime \prime}\right] \neq 0,
$$

and a singular point if $D(t)=0$. A singular point is called an ordinary point of inflection if in addition the derivative

$$
D^{\prime}(t)=\left[x x^{\prime} x^{\prime \prime \prime}\right] \neq 0 .
$$

We assume that any singular point of $\gamma$ is an ordinary point of inflection such that cusps are excluded. The curve may intersect itself at one or more double points.

If $\gamma$ lies in an affine plane the projective coordinates may be replaced by affine coordinates, where $x_{0}(t)=1$. Introduce the vector $r(t)=\left(x_{1}(t), x_{2}(t)\right)$. Then the point $P$ will be a convex point when $D(t)=\left[\boldsymbol{r}^{\prime} \boldsymbol{r}^{\prime \prime}\right] \neq 0$ and an ordinary point of inflection for

$$
D(t)=0 \quad \text { and } \quad D^{\prime}(t)=\left[\boldsymbol{r}^{\prime} \boldsymbol{r}^{\prime \prime \prime}\right] \neq 0 .
$$


3. First we consider the case where $\gamma$ is lying in an affine plane. The affine curvature $k=k(t)$ at the point $P$ is given by the formula

$$
k(t)=\frac{1}{9}\left[\boldsymbol{r}^{\prime} \boldsymbol{r}^{\prime \prime}\right]^{-\frac{8}{3}}\left(3\left[\boldsymbol{r}^{\prime} \boldsymbol{r}^{\prime \prime}\right]\left[\boldsymbol{r}^{\prime} \boldsymbol{r}^{\prime \prime \prime \prime}\right]+12\left[\boldsymbol{r}^{\prime} \boldsymbol{r}^{\prime \prime}\right]\left[\boldsymbol{r}^{\prime \prime} \boldsymbol{r}^{\prime \prime \prime}\right]-5\left[\boldsymbol{r}^{\prime} \boldsymbol{r}^{\prime \prime \prime}\right]^{2}\right)
$$

([2, p. 150]). It is seen that $k(t)$ exists and varies continuously when the point $P(t)$ traverses an arc between two consecutive points of inflection $P_{1}=P\left(t_{1}\right)$ and $P_{2}=P\left(t_{2}\right)$. Hence the function $k(t)$ has a maximum for some value $t=\tau \in] t_{1} ; t_{2}[$, and $P(\tau)$ is a sextactic point. Thus we have shown

THEOREM 1. When $\gamma$ lies in an affine plane there is at least one sextactic point between two consecutive points of inflection (fig. 1).

4. Now, let $\gamma$ lie in a projective plane and, as above, let $P_{1}$ and $P_{2}$ denote two consecutive ordinary points of inflection of $\gamma$. If the $\operatorname{arc} P_{1} P_{2}$ has no double point it has no singularities at all, and it is either a convex arc, a single or a double spiral [3, pp. 149-153]. In any case there are lines that have no point in common with the arc such that it may be considered as lying in an affine plane. Hence, applying theorem 1, we get

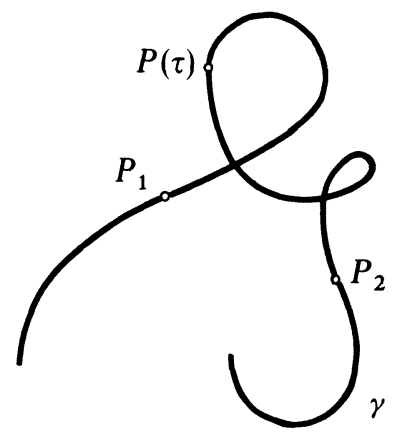

Fig. 1

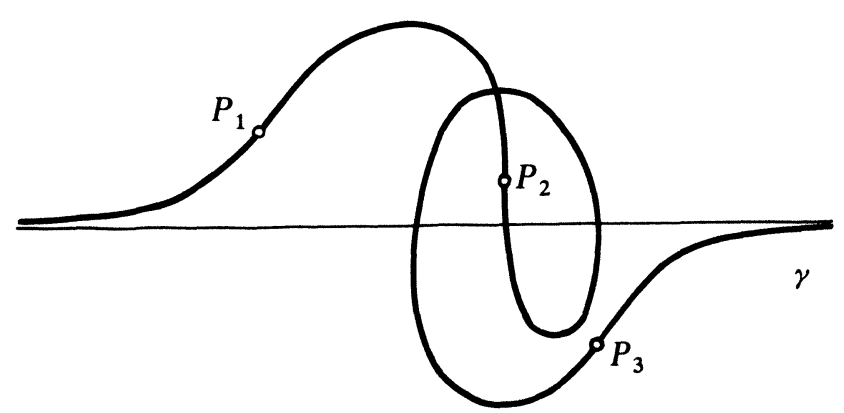

Fig. 2 
TheOREM 2. When $\gamma$ lies in a projective plane and the arc between two consecutive points of inflection has no double point, then there is at least one sextactic point on the arc.

More generally we can state

THEOREM 3. When $\gamma$ lies in a projective plane and has p points of inflection, and no arc connecting two consecutive points of inflection intersects itself, then $\gamma$ has at least $p-1$ or $p$ sextactic points according as the curve is open or closed.

The curve may have double points, but only when they are points of intersection between arcs connecting different points of inflection (fig. 2).

5. For a closed curve without double points and cusps Möbius has shown that it has at least three points of inflection (see e.g. [1, p. 42]). If the curve is of class $C^{4}$ and every singular points is an ordinary point of inflection, then theorem 3 may be applied, and it follows that the curve has at least three sextactic points. Thus we have proved the conjecture of G. Bol.

\section{REFERENCES}

1. G. Bol, Projective Differentialgeometrie vol. 1 (Studia Mathematica, Mathematische Lehrbücher 4), Vandenhoeck \& Ruprecht, Göttingen, 1950.

2. H. Guggenheimer, Differential geometry, McGraw-Hill, New York, 1963.

3. O. Haupt und H. Künneth, Geometrische Ordnungen, (Grundlehren Math. Wissensch. 133), Springer-Verlag, Berlin - New York, 1967.

DANMARKS TEKNISKE HØJSKOLE

LYNGBY

DENMARK 